\title{
Condições crônicas complexas e desfechos hospitalares em uma unidade de terapia intensiva pediátrica
}

Complex chronic conditions and hospital outcomes in a pediatric intensive care unit

Condiciones crónicas complejas y resultados hospitalarios en una unidad de cuidados intensivos pediátricos

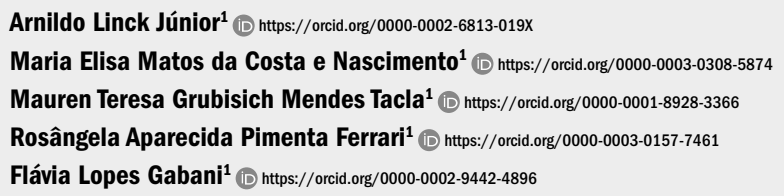

\section{Resumo}

Objetivo: Analisar associação das condições crônicas complexas (CCC) com óbito numa unidade de terapia intensiva pediátrica (UTIP). Secundariamente, verificar associação com suporte ventilatório invasivo e hemodinâmico, infecções hospitalares e tempo de internação.

Métodos: Coorte retrospectiva, entre 2012 e 2017, com internações de crianças com CCC, considerada variável de exposição. Utilizou-se regressão de Poisson, com significância de $5 \%$ e cálculo do risco relativo (RR).

Resultados: Das 585 crianças, 51,3\% tinham CCC. Foram mais frequentes encefalopatias, neoplasias e pneumopatias. Essas crianças também apresentaram menor risco para uso de drogas vasoativas (DVA) nas primeiras 24 horas de terapia intensiva $(\mathrm{RR}=0,72$; IC95\%=0,54-0,98), porém foi maior a incidência de infecções hospitalares (RR=1,49; IC95\%=1,10-2,03) e óbito (RR=2,14; IC95\%=1,46-3,13), mesmo após controle por variáveis de confusão.

Conclusão: Verificou-se elevada frequência de CCC, com diferença significativa para menor risco de uso de DVA nas primeiras 24 horas da internação, e maior incidência de infecções hospitalares e óbito.

\begin{abstract}
Objective: To analyze the association of complex chronic conditions (CCC) with death in a pediatric intensive care unit and association with hemodynamic and ventilatory support, hospital infections and length of stay. Methods: Retrospective cohort, between 2012 and 2017, with hospitalizations of children with CCC, considered an exposure variable. Poisson regression was used, with $5 \%$ significance and calculation of relative risk (RR).

Results: Of the 585 children, $51.3 \%$ had CCC. Encephalopathies, neoplasms and pneumopathies were more frequent. These children had lower risk of using vasoactive drugs (VAD in the first 24 hours of admission ( $R R=0.72$; $95 \% \mathrm{Cl}=0.54-0.98)$. The incidence of nosocomial infections was higher ( $\mathrm{RR}=1.49 ; 95 \% \mathrm{Cl}=1.10-2.03)$ and death $(\mathrm{RR}=2.14 ; 95 \% \mathrm{Cl}=1.46-3.13)$, even after controlling for confounding variables.

Conclusion: There was a high frequency of CCC, with a significant difference for a lower risk of using VAD in the first 24 hours of admission, and a higher incidence of hospital infections and death.

\section{Resumen}

Objetivo: Analizar la asociación de enfermedades crónicas complejas (CCC) con la muerte en una unidad de cuidados intensivos pediátricos (UCIP). En segundo lugar, verificar la asociación con soporte ventilatorio invasivo y hemodinámico, infecciones hospitalarias y duración de la estancia.

Métodos: Cohorte retrospectiva, entre 2012 y 2017, con hospitalizaciones de niños con CCC, considerada variable de exposición. Se utilizó regresión de Poisson, con $5 \%$ de significación y cálculo del riesgo relativo (RR).

Resultados: De los 585 niños, el 51,3\% tenía CCC. Las encefalopatías, neoplasias y neumopatías fueron más frecuentes. Estos niños también tenían un menor riesgo de usar fármacos vasoactivos (DAV) en las primeras 24 horas de cuidados intensivos ( $R R=0,72$; IC95\% $=0,54-0,98$ ), pero la incidencia de infecciones nosocomiales fue mayor $(\mathrm{RR}=1,49 ;$ IC95\% = 1,10-2,03) y muerte $(\mathrm{RR}=2,14 ;$ IC95\% = 1,46-3,13), incluso después de controlar las variables de confusión.

Conclusión: Hubo una alta frecuencia de CCC, con una diferencia significativa para un menor riesgo de uso de DAV en las primeras 24 horas de ingreso, y una mayor incidencia de infecciones hospitalarias y muerte.
\end{abstract}

\section{Como citar:}

Linck Júnior A, Nascimento ME, Tacla MT, Ferrari RA, Gabani FL. Condições crônicas complexas e desfechos hospitalares em uma unidade de terapia intensiva pediátrica. Rev Soc Bras Enferm Ped. 2021;21(2):125-32.

\footnotetext{
${ }^{1}$ Universidade Estadual de Londrina, Londrina, PR, Brasil.

Conflitos de interesse: nada a declarar.

Submetido: 25 de Agosto de 2021 | Aceito: 3 de Dezembro de 2021

Autor correspondente: Flávia Lopes Gabani | E-mail: Iopesgabani@gmail.com

Dol: http://dx.doi.org/10.31508/1676-379320210018
}

\section{Descritores \\ Doença crônica; Unidades de terapia intensiva pediátrica; Mortalidade hospitalar; Saúde da criança; Enfermagem pediátrica}

\section{Keywords}

Chronic disease; Intensive care units pediatric; Hospital mortality; Child health; Pediatric nursing

\section{Descriptores}

Enfermedad crónica; Unidades de cuidado intensivo pediátrico; Mortalidad hospitalaria; Salud del niño; Enfermería pediátrica 


\section{Introdução}

As doenças crônicas são geralmente multifatoriais e de início gradual, com longa ou indefinida duração, podendo gerar potenciais incapacidades..$^{(1)}$ No caso de crianças, essas doenças, consideradas como condições crônicas complexas (CCC), ${ }^{(2)}$ causam prejuízos à saúde mental e desenvolvimento pelo diagnóstico em si, e por maior exposição à dor, realização frequente de exames e maior ocorrência de internações. Também, há limitação de atividades inerentes à infância, como frequentar escola, brincar, entre outros hábitos infantis socialmente saudáveis. ${ }^{(3)}$ Para a família, esse diagnóstico gera rompimento da rotina, com alteração do cotidiano para centrar o cuidado à criança, o que pode desencadear instabilidade emocional e estado de vulnerabilidade. ${ }^{(4)}$

A discussão sobre prevalência e consequências das CCC ganha dimensão ainda maior na área pediátrica ao se considerar impacto nos ambientes hospitalares. ${ }^{(5,6)}$ Maior disponibilidade de leitos de terapia intensiva, avanço tecnológico e aprimoramento de recursos humanos, principalmente em unidades neonatais, vêm permitindo sobrevida de crianças graves, com diagnósticos de malformações e extremamente prematuras, sobrecarregando sistemas de saúde com reinternações e reabilitações. ${ }^{(7)}$

Pesquisa realizada em hospital público federal especializado em assistência pediátrica, no município do Rio de Janeiro, comparou pacientes com e sem CCC. O objetivo foi identificar a utilização de tecnologias, e mensurar custo da assistência hospitalar dessas crianças e adolescentes. Como resultados verificaram que pacientes com CCC internaram, em média, duas vezes ao ano, e tiveram maior necessidade de uso de dispositivos hospitalares, como drenos, cateteres venosos e gastrostomia, tornando a estadia hospitalar mais dispendiosa. ${ }^{(8)}$

Estudo longitudinal, realizado no Reino Unido, buscou mensurar prevalência e impacto das condições crônicas em relação ao tempo de internação e morbimortalidade em unidade de terapia intensiva pediátrica (UTIP). Das 1197 crianças de zero a 18 anos, admitidas entre 2009 e 2013, 554 (46,3\%) tinham pelo menos uma condição crônica, avaliadas como cromossomopatias, cardiovasculares, respiratórias, gastrointestinais, hematológicas, neurológicas e endócrinas. Essas crianças apresentaram maior tempo de internação e óbito em relação às demais. ${ }^{(9)}$

Dessa forma, são crianças que demandam leitos de terapia intensiva, com maior risco de complicações, necessidade de suporte ventilatório, maior risco de infecções hospitalares decorrentes de manipulações excessivas, imunossupressão e estadia hospitalar prolongada, além de maior risco de óbito em relação às demais. ${ }^{(5,10,11)}$

Conhecer o impacto das CCC em ambientes de cuidados intensivos pode colaborar para o alcance de estratégias que visem menos hospitalizações e melhora da qualidade de sobrevida. O principal objetivo deste estudo foi analisar a associação das CCC com o óbito em crianças internadas em uma UTIP de referência no norte do Paraná, Brasil. Como objetivos secundários, verificar a associação dessas condições com necessidade de suporte ventilatório invasivo e hemodinâmico, infecções hospitalares e tempo de internação. Essa compreensão pode promover estratégias para maior rotatividade de leitos, proporcionando mais acessibilidade à população pediátrica portadora de doenças graves.

\section{Métodos}

Trata-se de um estudo de coorte retrospectiva, realizado com internações de crianças admitidas em uma UTIP de hospital universitário localizado em município do norte do Estado do Paraná, Sul do Brasil, durante os anos de 2012 (1ํ de janeiro) a 2017 (31 de dezembro). Esse hospital é considerado de alta complexidade, atendendo pacientes de cerca de $250 \mathrm{mu}-$ nicípios do Paraná, e de mais de 100 cidades de outros Estados. A UTIP pesquisada é classificada como mista, ou seja, admite desde recém-nascidos até crianças com 15 anos incompletos, e é considerada referência para atendimentos especializados, como traumas, nefropediatria, cirurgia infantil, neurocirurgia e queimaduras graves. Além disso, é a única da cidade com atendimento exclusivo pelo Sistema Único de Saúde (SUS), comportando, até o final do período deste estudo, sete leitos.

Foram consideradas para a pesquisa todas as internações de crianças no período supracitado, que tinham diagnóstico de CCC em prontuário médi- 
co. Para Feudtner et al. ${ }^{(12)}$ e Feudtner, Christakis and Connell(2) são doenças que cursam com expectativa de sobrevida de no mínimo 1 ano, e que acometem diferentes órgãos e sistemas ou um órgão de forma intensa suficiente para que o paciente necessite de atendimento pediátrico especializado, e provavelmente um período de hospitalização em um centro terciário. Esse conceito também é reforçado pelo Centers for Disease Control and Prevention (CDC), o qual considera como condições que duram um ano ou mais e requerem atendimento médico contínuo ou trazem limitações à vida do indivíduo.(13) Para adequação da população foram excluídas internações de crianças em que essa condição diagnóstica era ignorada, e àquelas com menos de um ano de idade, contemplando neste estudo as idades de um a 15 anos, mais um adolescente de 18 anos admitido no período analisado por apresentar apenas 17 quilos.

A coleta de dados ocorreu por meio da análise de prontuários dos pacientes que foram internados na UTIP, disponibilizados pelo Serviço de Arquivo Médico e Estatística (SAME) do hospital. Foi utilizado instrumento estruturado em sete blocos: (1) caracterização social e demográfica, (2) antecedentes da criança, (3) admissão na UTIP, (4) avaliação inicial, (5) internação na UTIP, (6) ventilação pulmonar mecânica, e (7) dados sobre o óbito. Para este estudo, foram consideradas as seguintes variáveis: diagnóstico de CCC (sim e não), classificação da CCC (cardiopatia, cromossomopatia, doença hematológica, encefalopatia, endocrinopatia, gastroenteropatia, hepatopatia, malformações, nefropatia, neoplasia e pneumopatia), número de CCC, sexo (masculino e feminino), idade em anos (1 a 3, 4 a 9, >9), procedência (próprio município e outros municípios), e diagnóstico de admissão conforme capítulo da Classificação Internacional de Doenças, décima revisão (CID-10). A CCC foi considerada variável de exposição para os seguintes desfechos: necessidade de ventilação pulmonar mecânica-VPM (sim e não), tempo de VPM (conforme mediana das crianças com e sem (CC), necessidade de drogas vasoativas-DVA nas primeiras 24 horas (sim e não), tempo de uso de DVA (conforme mediana das crianças com e sem (CC), infecções relacionadas à assistência à saúde-IRAS (sim e não), diagnósticos de infecção fúngica (sim e não), e infecção de corrente sanguínea relacionada a cateter-ICSRC (sim e não), tempo de internação em
UTIP e hospitalar, em dias, conforme mediana das populações com e sem CCC, e óbito (sim e não).

Os dados coletados foram digitados no programa Epi Info ${ }^{\circledR}$ e analisados no programa Statistical Package for the Social Sciences $\left(S P S S^{\circledR}\right)$, versão 19. Para análise estatística considerou-se nível de significância de 5\%, com cálculo da razão de prevalência (RP) para as variáveis admissionais (sexo, idade e procedência), e cálculo do risco relativo (RR) para análise entre variável de exposição e desfechos, ambos com intervalo de confiança (IC 95\%). Foi utilizado modelo de regressão de Poisson com variância robusta para as análises de associação, o qual é recomendado quando o desfecho é dicotômico e tem frequência elevada. Para análises significativas entre fator (CCC) e desfecho, o RR foi ajustado por variáveis que se associaram aos desfechos com valor de $p<0,20$.

Essa pesquisa seguiu as normas referentes à Resolução no 510/2016, e obteve aprovação do Comitê de Ética em Pesquisa Envolvendo Seres Humanos da instituição (CAAE: 83069418.7.0000.5231).

\section{Resultados}

Entre 2012 e 2017 ocorreram 1.223 admissões, em que 41 foram consideradas perdas por falta de acesso aos prontuários, restando 1.182 internações. Dessas, excluíram-se 570 por serem crianças menores de um ano, e 27 por não apresentar registro sobre CCC em prontuário, totalizando para este estudo população final de 585 . A prevalência de CCC durante os seis anos analisados foi 51,3\% (300), com variações ao longo do período: 2012 (50,0\%), 2013 (60,6\%), 2014 (58,8\%), 2015 (40,0\%), 2016 (48,4\%); 2017 (50,0\%). A mediana da idade na população geral foi 59 meses, sendo 62,5 meses entre crianças com CCC, e 49 meses nas demais. A tabela 1 apresenta as características demográficas segundo presença de CCC. A prevalência de CCC foi maior entre crianças do sexo feminino ( $R P=1,19$; IC $95 \%=1,02-1,40)$, sem diferença estatística quanto à faixa etária e procedência.

Verificou-se prevalência de encefalopatias (107; $28,7 \%)$, neoplasias $(64 ; 17,7 \%)$ e pneumopatias (50; $13,4 \%)$. Nesta população, as cardiopatias representaram apenas 5,9\% (22) dos diagnósticos (Figura 1). Grande parte das crianças apresentavam apenas uma CCC $(240 ; 80,0 \%)$, seguido por duas $(50 ; 16,7 \%)$ e três (10; $3,3 \%)$, com média de 1,23 por criança. 
Tabela 1. Características demográficas segundo presença de condição crônica complexa em internações de uma unidade de terapia intensiva pediátrica (UTIP)

\begin{tabular}{|c|c|c|c|c|}
\hline \multirow[b]{2}{*}{ Variáveis } & \multirow[b]{2}{*}{$\mathrm{n}(\%)$} & \multicolumn{3}{|c|}{ Condição crônica complexa } \\
\hline & & $\begin{array}{l}\text { Sim } \\
\mathrm{n}(\%)\end{array}$ & RP (IC 95\%) & $p$-value \\
\hline \multicolumn{5}{|l|}{ Sexo* } \\
\hline Masculino & $307(52,6)$ & $144(46,9)$ & 1 & \\
\hline Feminino & $277(47,4)$ & $155(56,0)$ & $\begin{array}{c}1,19 \\
(1,02-1,40)\end{array}$ & 0,029 \\
\hline \multicolumn{5}{|l|}{ Idade (anos) } \\
\hline 1 a 3 & $252(43,1)$ & $114(45,2)$ & $\begin{array}{c}1,06 \\
(0,98-1,13)\end{array}$ & 0,131 \\
\hline 4 a 9 & $198(33,8)$ & $114(57,6)$ & $\begin{array}{c}0,97 \\
(0,90-1,05)\end{array}$ & 0,443 \\
\hline$>9$ & $135(23,1)$ & $72(53,3)$ & 1 & \\
\hline \multicolumn{5}{|l|}{ Procedência* } \\
\hline Outros municípios & $349(59,8)$ & $177(50,7)$ & $\begin{array}{c}1,01 \\
(0,96-1,07)\end{array}$ & 0,700 \\
\hline Próprio município & $235(40,2)$ & $123(52,3)$ & 1 & \\
\hline
\end{tabular}

Quanto aos principais diagnósticos admissionais, destacaram-se as doenças do aparelho respiratório $(23,7 \%)$, neoplasias $(17,7 \%)$ e malformações congênitas, deformidades e anomalias cromossômicas $(9,0 \%)$ (Figura 2).

Em relação aos desfechos analisados (Tabela 2), crianças com diagnóstico de CCC apresentaram menor risco para uso de DVA nas primeiras 24 horas ( $R R=0,73 ;$ IC 95\%=0,55-0,98). Porém, diagnóstico de IRAS (RR=1,47; IC95\%=1,07-2,03) e óbito ( $R R=1,83$; IC95\%=1,21-2,79) foram mais incidentes nessa população. Não houve diferença estatística significativa nas demais variáveis analisadas, apesar de crianças com CCC apresentarem maiores frequências de permanência na VPM por 5 dias ou mais, diagnósticos de infecção fúngica e ICSRC, e internação na UTIP por

*Excluídos registros com informações ignoradas.

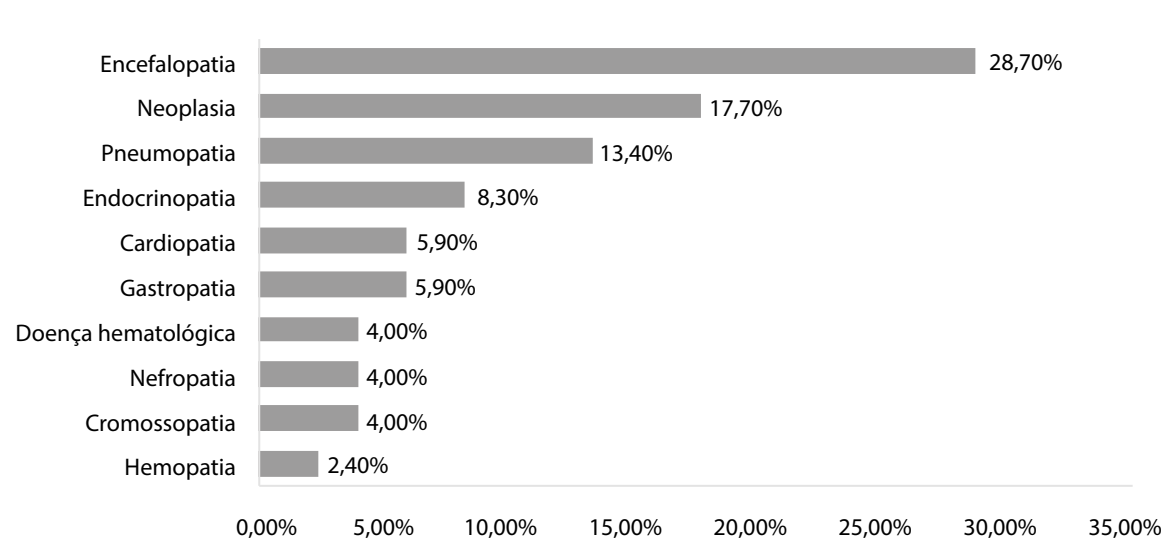

Figura 1. Classificação das principais condições crônicas complexas identificadas nas admissões de uma unidade de terapia intensiva pediátrica (UTIP) $(n=370)$

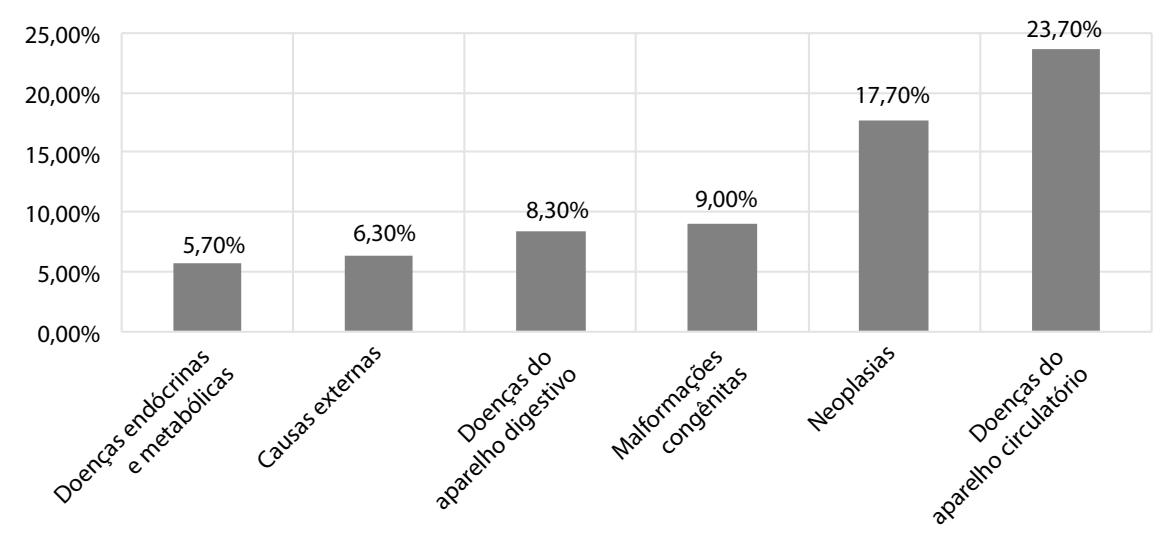

Figura 2. Distribuição do diagnóstico principal de admissão das internações de crianças com condições crônicas complexas de uma unidade de terapia intensiva pediátrica (UTIP) 
Tabela 2. Desfechos das internações de crianças com condição crônica complexa em uma unidade de terapia intensiva pediátrica (UTIP)

\begin{tabular}{|c|c|c|c|c|}
\hline \multirow{2}{*}{ Desfechos da internação } & \multicolumn{2}{|c|}{$\begin{array}{l}\text { Condição crônica } \\
\text { complexa }\end{array}$} & \multirow{2}{*}{$\begin{array}{l}\mathrm{RR}(\mathrm{IC} \\
95 \%)\end{array}$} & \multirow{2}{*}{$p$-value } \\
\hline & $\begin{array}{l}\text { Sim } \\
\mathrm{n}(\%)\end{array}$ & $\begin{array}{l}\text { Não } \\
\mathrm{n}(\%)\end{array}$ & & \\
\hline \multicolumn{5}{|l|}{ Necessidade de VPM } \\
\hline $\operatorname{Sim}$ & $163(54,3)$ & $153(53,7)$ & $\begin{array}{c}1,01 \\
(0,87-1,18)\end{array}$ & 0,875 \\
\hline Não & $137(45,7)$ & $132(46,3)$ & 1 & \\
\hline \multicolumn{5}{|l|}{ Tempo de VPM (dias) ${ }^{\mp}$} \\
\hline Até 4 & $92(53,5)$ & $91(59,1)$ & 1 & \\
\hline 5 e mais & $80(46,5)$ & $63(40,9)$ & $\begin{array}{c}1,14 \\
(0,89-1,46)\end{array}$ & 0,311 \\
\hline \multicolumn{5}{|l|}{ Necessidade de DVA* } \\
\hline $\operatorname{Sim}$ & $61(20,3)$ & $79(27,8)$ & $\begin{array}{c}0,73 \\
(0,55-0,98)\end{array}$ & 0,035 \\
\hline Não & $239(79,9)$ & $205(72,2)$ & 1 & \\
\hline \multicolumn{5}{|l|}{ Tempo de DVA (dias) ${ }^{\mp}$} \\
\hline Até 3 & $53(64,6)$ & $48(56,5)$ & 1 & \\
\hline 4 e mais & $29(35,4)$ & $37(43,5)$ & $\begin{array}{c}0,81 \\
(0,56-1,19)\end{array}$ & 0,284 \\
\hline \multicolumn{5}{|l|}{ IRAS } \\
\hline $\operatorname{Sim}$ & $76(25,3)$ & $49(17,2)$ & $\begin{array}{c}1,47 \\
(1,07-2,03)\end{array}$ & 0,018 \\
\hline Não & $224(74,7)$ & $236(82,8)$ & 1 & \\
\hline \multicolumn{5}{|l|}{ Infecção fúngica } \\
\hline $\operatorname{Sim}$ & $38(12,7)$ & $25(8,8)$ & $\begin{array}{c}1,44 \\
(0,90-2,33)\end{array}$ & 0,122 \\
\hline Não & $262(87,3)$ & $260(91,2)$ & 1 & \\
\hline \multicolumn{5}{|l|}{ ICSRC } \\
\hline $\operatorname{Sim}$ & $17(5,7)$ & $9(3,2)$ & $\begin{array}{c}1,79 \\
(0,81-3,96)\end{array}$ & 0,148 \\
\hline Não & $283(94,3)$ & $276(96,8)$ & 1 & \\
\hline \multicolumn{5}{|l|}{$\begin{array}{l}\text { Tempo de internação na } \\
\text { UTIP (dias) }{ }^{\mp}\end{array}$} \\
\hline Até 3 & $163(54,3)$ & $176(61,8)$ & 1 & \\
\hline 4 e mais & $137(45,7)$ & $109(38,2)$ & $\begin{array}{c}1,19 \\
(0,99-1,45)\end{array}$ & 0,071 \\
\hline \multicolumn{5}{|l|}{$\begin{array}{l}\text { Tempo de internação } \\
\text { hospitalar (dias) }^{\mp}\end{array}$} \\
\hline Até 9 & $165(55,0)$ & $138(48,4)$ & 1 & \\
\hline 10 e mais & $135(45,0)$ & $147(51,6)$ & $\begin{array}{c}0,87 \\
(0,74-1,03)\end{array}$ & 0,112 \\
\hline \multicolumn{5}{|l|}{ Óbito } \\
\hline $\operatorname{Sim}$ & $56(18,7)$ & $29(10,2)$ & $\begin{array}{c}1,83 \\
(1,21-2,79)\end{array}$ & 0,004 \\
\hline Não & $244(81,3)$ & $256(89,8)$ & 1 & \\
\hline
\end{tabular}

*Excluídos registros com informações ignoradas. VPM: ventilação pulmonar mecânica. DVA: droga vasoativa. ${ }^{\mp} M e d i a n a$ na população geral. IRAS: infecções relacionadas à assistência à saúde. ICSRC: infecção de corrente sanguínea relacionada a cateter.

quatro ou mais dias. O menor risco para uso de DVA manteve-se significativo após ajuste por idade e anemia $(R R=0,72 ; \mathrm{IC} 95 \%=0,54-0,98 ; \mathrm{p}=0,035)$.

As medianas entre crianças com e sem CCC foram semelhantes nas variáveis tempo de VPM (4 a 3 dias, respectivamente), tempo de DVA (2,5 e 3 dias, respec- tivamente), tempo de internação da UTIP (3 e 2 dias, respectivamente) e tempo de internação hospitalar ( 9 e 10 dias, respectivamente). Na tabela 3 observa-se que os desfechos IRAS (RR=1,49; IC95\%=1,10-2,03) e óbito $(R R=2,14$; IC95\%=1,46-3,13) permaneceram significativos mesmo após ajustes progressivos por variáveis de confusão.

Tabelas 3. Risco relativo (RR) bruto e ajustado e intervalo de confiança de $95 \%$ para desfechos em crianças com condições crônicas complexas de uma unidade de terapia intensiva pediátrica (UTIP)

\begin{tabular}{l|ccc}
\hline Desfechos & $\begin{array}{c}\text { Análise bruta } \\
\text { RR (IC95\%) }\end{array}$ & $\begin{array}{c}\text { Análise ajustada } \\
\text { (Modelo 1) } \\
\text { RR (IC95\%) }\end{array}$ & $\begin{array}{c}\text { Análise ajustada } \\
\text { (Modelo 2) } \\
\text { RR (IC95\%) }\end{array}$ \\
\hline IRAS & $1,47(1,07-2,03)$ & $1,49(1,08-2,05)^{*}$ & $1,49(1,10-2,03)^{\mp}$ \\
Óbito & $1,83(1,21-2,79)$ & $1,81(1,20-2,74)^{\ddagger}$ & $2,14(1,46-3,13)^{\mp}$ \\
\hline
\end{tabular}

IRAS - infecções relacionadas à assistência à saúde; "Ajustado por idade (variável categórica), sexo e procedência; ${ }^{\mp}$ Modelo 1 , necessidade de VPM e necessidade de DVA; ${ }^{\mathrm{t}}$ Ajustado por sexo e procedência

\section{Discussão}

Este estudo identificou que $51,3 \%$ das crianças internadas na UTIP possuíam uma ou mais CCC, com maior ocorrência no sexo feminino. Prevaleceram as encefalopatias, seguidas pelas neoplasias e pneumopatias. As doenças do aparelho respiratório foram os diagnósticos admissionais mais frequentes. Crianças com CCC apresentaram menor risco para necessidade de uso de DVA nas primeiras 24 horas de terapia intensiva, por outro lado foi maior a incidência de IRAS e óbito nessa população, mesmo após controle por variáveis de confusão. Os demais desfechos analisados não resultaram em significância estatística, apesar da permanência no VPM por 5 dias ou mais, diagnósticos de infecção fúngica e ICSRC, e internação na UTIP por quatro ou mais dias apresentarem maiores proporções no grupo de CCC.

O número de pacientes com doenças crônicas tem aumentado nos últimos 20 anos, porém dados sobre a prevalência na população pediátrica e seu impacto nas internações hospitalares são limitados, principalmente no Brasil. Penela-Sánchez et al. ${ }^{(14)}$ identificaram em hospital da Espanha que 14,4\% das internações pediátricas foram de pacientes portadores de condições complexas crônicas. Percentuais diferentes desse estudo, e semelhantes à presente pesquisa, foram identificados em populações de crianças graves nos Estados 
Unidos $(40,0 \% \text { a } 53,0 \%)^{(10,11)}$ e Argentina $(48,1 \%){ }^{(5)}$ possivelmente pela criança portadora de patologia crônica normalmente apresentar pior evolução clínica e necessidade de suporte intensivo.

Outra característica deste estudo é a maior prevalência de admissões de crianças do sexo feminino portadoras de CCC, diferente do observado em pesquisa realizada nos Estados Unidos. ${ }^{(10)} \mathrm{A}$ maior mediana de idade entre essas crianças justifica-se pela progressão da doença de base em pacientes mais velhos, resultando em internações e reinternações mais frequentes. ${ }^{(14)}$

Quanto à classificação das principais CCC, destacaram-se as encefalopatias, seguidas pelas neoplasias e pneumopatias. López et al. ${ }^{(5)}$ identificaram predomínio de doenças cardiovasculares $(22,24 \%)$, neuromusculares (18,75\%) e doença maligna (17,7\%) em UTIP na Argentina. Apesar das semelhanças em alguns grupos mais frequentes, a variação da proporção pode ocorrer pelas particularidades do serviço de saúde desta pesquisa, em que neuropediatria e neurocirurgia são referências regionais para atendimento destes pacientes. Por outro lado, existem hospitais no município que concentram atendimentos de crianças cardiopatas e oncológicas, diluindo as demandas dessas CCC entre esses serviços assistenciais. Destaca-se, porém, que as neoplasias atendidas na UTIP deste estudo concentram-se em tumores no sistema nervoso central, em que há necessidade de abordagens cirúrgicas pela equipe especializada do município e, após a recuperação do pós-operatório, essas crianças são contrarreferenciadas ao centro de atendimento especializado para continuidade do tratamento.

A média de CCC deste estudo foi de 1,23 por criança, em que apenas $20,0 \%$ tinham duas ou mais. Segundo Nadeau et al.(15) $43 \%$ das crianças admitidas em uma UTIP norte-americana tinham mais de uma condição crônica complexa. Isso é inerente da própria definição, em que o diagnóstico limitador das condições de vida pode envolver mais de um órgão ou sistema, ou apenas um órgão de forma severa, com possibilidade de repercutir negativamente sobre outros. ${ }^{(12)}$

Doenças respiratórias foram os principais diagnósticos admissionais de crianças com CCC. Estudo da Nova Zelândia descreveu os fatores de risco para readmissão após internação hospitalar por doenças infecciosas em menores de dois anos, identificando que das 46.657 crianças avaliadas, 10.205 (22\%) tiveram pelo menos uma readmissão por doença infecciosa em até 12 meses, tendo como causa principal doenças respiratórias. Entre os fatores de risco reportados, destacou-se presença de pelo menos uma CCC. ${ }^{(16)}$ Em outra UTIP, admissões por pós-operatório e doenças respiratórias foram as mais frequentes entre esses pacientes. ${ }^{(5)}$ Em UTIP americanas, prevaleceram problemas respiratórios, seguidos pelos neurológicos. ${ }^{(10)}$ Apesar da diversidade das CCC limitadoras de vida, normalmente as encefalopatias, condição mais prevalente entre as admissões desta pesquisa, cursam com acometimento simultâneo do sistema respiratório, agravando ainda mais a fragilidade pulmonar intrínseca da criança e repercutindo nos índices de morbidade por essa causa.

Quantos aos desfechos, crianças com CCC apresentaram menor risco para uso de DVA nas primeiras 24 horas da internação, independente da faixa etária e diagnóstico de anemia. Por se tratar de comorbidades complexas, as admissões podem representar estado mais crítico de evolução, com necessidade de suporte hemodinâmico, contudo, a incorporação de práticas de cuidados paliativos, ainda que de forma incipiente, pode ter refletido na não introdução desse suporte em portadores de CCC. Esta pesquisa não mensurou escore de gravidade na admissão, sendo essa, portanto, uma hipótese de associação.

A incidência de IRAS e óbito foi maior na população com CCC. Os demais desfechos analisados não apresentaram significância estatística, apesar da permanência no VPM por 5 dias ou mais, diagnósticos de infecção fúngica e ICSRC, e internação na UTIP por quatro dias ou mais terem maiores proporções nesse grupo. Pacientes com condições crônicas requerem internação prolongada, ${ }^{(5,10,11,17)}$ maior necessidade de cuidados intensivos ${ }^{(17)}$ e uso de tecnologia como, por exemplo, suporte ventilatório. ${ }^{(5)}$

No Reino Unido, estudo apontou que o tempo de internação hospitalar para pacientes com pelo menos uma condição crônica foi, em média, quatro dias. Em casos de três ou mais, este tempo aumentava para 5,4 dias. Também houve aumento da mortalidade em pacientes com duas ou mais condições crônicas comparados com aqueles sem esse diagnóstico. ${ }^{(9)}$ Portanto, trata-se de condição que predispõe a criança ao maior risco de óbito dentro das UTIP. ${ }^{(10)}$ Pesquisa na Holanda que investigou fatores associados à mortalidade em 
pacientes pediátricos de terapia intensiva com baixo risco de óbito identificou condições crônicas como fatores significativamente associados $(76,7 \%$; OR=3,29; $95 \%$ CI, 1,97-5,50). ${ }^{(18)}$

Na presente pesquisa, além da maior incidência de óbitos, também houve maior frequência do diagnóstico de IRAS entre as crianças com CCC. Além do risco de um eventual estado de imunodeficiência, crianças com essas condições são dependentes de múltiplos cuidados assistenciais, de medicamentos para uso contínuo a necessidade de dispositivos, por exemplo, cânulas de traqueostomia, cateteres de gastrostomia, entre outros. ${ }^{(8)}$ Essas pré-condições aumentam o risco do desenvolvimento de infecções durante o período de hospitalização, notadamente naqueles com internação prolongada. ${ }^{(5)}$ Revisão integrativa publicada em 2017, ao analisar a incidência de infecções primárias de corrente sanguínea, uma das vertentes das IRAS, em uma população de adultos não neutropênicos, associou a condição de doença crônica com maiores tempos de hospitalização, bem como maior necessidade de antimicrobianos, consequentemente, maiores incidências de infecções adquiridas no ambiente hospitalar. ${ }^{(19)}$

A condução de CCC é desafiadora e divergente entre os profissionais de saúde. Apesar do avanço das discussões sobre cuidados paliativos na infância, muitas crianças crônicas com doenças incompatíveis com a vida acabam recebendo suporte terapêutico curativista. Isso prolonga suas vidas de forma artificial, expondo-as a riscos e desfechos semelhantes a outras crianças não portadoras dessa condição, ${ }^{(20)}$ além de elevarem gastos em saúde sem necessariamente promoverem qualidade de vida. ${ }^{(21)}$

Este estudo sugere algumas limitações. Os resultados refletem a realidade de apenas uma UTIP que concentra CCC específicas. Pesquisa multicêntrica, englobando hospitais referenciados para demais CCC, poderia esclarecer melhor o comportamento sobre desfechos em saúde nas UTIP. Também, o caráter retrospectivo da coorte pode ter prejudicado a consistência de algumas informações pela limitação de pesquisas em prontuários. Por fim, a definição de doença crônica é divergente na literatura, ${ }^{(22)}$ o que dificulta melhor comparabilidade dos resultados com outras pesquisas.

Esse estudo vem ampliar a análise dessas associações dentro do ambiente de terapia intensiva, por meio de estudo longitudinal, ideal para reportar causalidade dos fatos, tendo uma população pediátrica robusta. Sugere-se a realização de estudos prospectivos com inclusão de recém-nascidos e lactentes, em que é possível prever o diagnóstico de condição crônica limitadora de vida. Também, recomenda-se reflexão sobre a condução clínica dessas crianças dentro dos setores hospitalares, considerando o avanço da discussão sobre cuidados paliativos pediátricos no Brasil, com objetivo de qualificar a assistência e promover conforto por meio individualização do cuidado, sem causar distanásia e perpetuação do sofrimento.

\section{Conclusão}

Apesar de não ter sido observada associação entre CCC e tempo de internação ou ventilação mecânica invasiva, os resultados deste estudo indicam que esse diagnóstico pode aumentar a incidência de infecções relacionadas à assistência à saúde e a mortalidade. Instituir a assistência pormenorizada a crianças portadora de CCC, ao reduzir a incidência de IRAS e aumentar a chance de sobrevida, pode minimizar as internações mais prolongadas na UTIP e no hospital, contribuindo para maior rotatividade de leitos de UTIP, o que pode minimizar custos aos serviços de saúde de alta complexidade, sobretudo em hospital cuja abrangência ultrapassa os limites regionais e da macrorregião.

\section{Colaborações}

Linck Júnior A e Gabani FL participaram da idealização do projeto, análise dos dados, redação do manuscrito e aprovação da versão final. Nascimento MEMC participou da idealização do projeto, análise dos dados e redação do manuscrito. Tacla MTGM e Ferrari RAP participaram da redação do manuscrito e aprovação da versão final.

\section{Referências}

1. Brasil. Ministério da Saúde. Diretrizes e recomendações para o cuidado integral de doenças crônicas não-transmissiviveis: promoção da saúde, vigilância, prevenção e assistência. Brasília (DF): Ministério da Saúde; 2008 [citado 2021 Jun 20]. Available from: https:// bvsms.saude.gov.br/bvs/publicacoes/diretrizes_recomendacoes_cuidado_doencas_ cronicas.pdf 
2. Feudtner $C$, Christakis DA, Connell FA. Pediatric deaths attributable to complex chronic conditions: a population-based study of Washington State, 1980-1997. Pediatrics. 2000;106(Supplement 1):205-9.

3. Silva AC. Impacto da doença crônica infantil sobre a saúde mental de cuidadores primários e 0 desenvolvimento socioemocional da criança (dissertação). Salvador: Universidade Federal da Bahia; 2018.

4. Pinto MM, Coutinho SE, Collet N. Doença crônica na infância e a atenção dos serviços de saúde. Ciênc Cuid Saúde. 2016;15(3):498-506.

5. López MP, Fernández AL, Fiquepron K, Meregalli C, Ratto ME, Serrate AS. Prevalence of children with complex chronic conditions in PICUs of Argentina: a prospective multicenter study. Pediatr Crit Care Med. 2020;21(3):e143-e51.

6. Gold JM, Hall M, Shah SS, Thomson J, Subramony A, Mahant S, et al. Long length of hospital stay in children with medical complexity. J Hosp Med. 2016;11(11):750-6.

7. Moreira ME. Problemas de saúde na infância: desafios do tratamento à prevenção. Ciênc Saude Colet. 2010;15(2):318-9.

8. Pinto M, Gomes R, Tanabe RF, Costa AC, Moreira MC. Análise de custo da assistência de crianças e adolescentes com condições crônicas complexas. Ciênc Saude Colet. 2019;24(11):4043-52.

9. O'Brien S, Nadel S, Almossawi 0 , Inwald DP. The impact of chronic health conditions on length of stay and mortality in a general PICU. Pediatr Crit Care Med. 2017;18(1):1-7.

10. Edwards JD, Houtrow AJ, Vasilevskis EE, Rehm RS, Markovitz BP, Graham RJ, et al. Chronic conditions among children admitted to U.S. pediatric intensive care units: their prevalence and impact on risk for mortality and prolonged length of stay. Crit Care Med. 2012;40(7):2196-203.

11. O'Keefe S, Maddux AB, Bennett KS, Youngwerth J, Czaja AS. Variation in Pediatric Palliative Care Allocation Among Critically III Children in the United States. Pediatr Crit Care Med. 2021;22(5):462-73

12. Feudtner C, Feinstein JA, Satchell M, Zhao H, Kang TI. Shifting place of death among children with complex chronic conditions in the United States, 1989-2003. JAMA. 2007;297(24):2725-32.
13. Centers for Disease Control and Prevention (CDC).About chronic diseases. USA: CDC; 2020 [cited 202110 Jun]. Available from: https://www.cdc.gov/chronicdisease/about/index. $\mathrm{htm}$.

14. Penela-Sánchez D, Ricart S, Vidiella N, García-García JJ.A study of paediatric patients with complex chronic conditions admitted to a paediatric department over a 12 month period. Anal Pediatr. 2020; S1695-4033(20)30287-3.

15. Nadeau N, Monuteaux MC, Tripathi J, Stack AM, Perron C, Neuman MI. Pediatric ICU transfers within 24 hours of admission from the emergency department: rate of transfer, outcomes, and clinical characteristics. Hosp Pediatr. 2019;9(5):393-7.

16. Seibt S, Gilchrist CA, Reed PW, Best EJ, Harnden A, Camargo CA, et al. Hospital readmissions with acute infectious diseases in New Zealand children $<2$ years of age. BMC Pediatr. 2018;18(1):1-9.

17. Penela-Sánchez D, Ricart S, Vidiella N, García-García JJ. Estudio de los pacientes pediátricos crónicos complejos ingresados en un senvicio de pediatría a lo largo de 12 meses. Anal Pediatr. 2020;95(4):233-9.

18. Verlaat CW, Visser IH, Wubben N, Hazelzet JA, Lemson J, Van Waardenburg D, et al. Factors associated with mortality in low-risk pediatric critical care patients in the Netherlands. Pediatr Crit Care Med. 2017;18(4):e155-e61.

19. Calik Basaran N, Ascioglu S. Epidemiology and management of healthcare-associated bloodstream infections in non-neutropenic immunosuppressed patients: a review of the literature. Ther Adv Infect Dis. 2017;4(6):171-91.

20. Ramelet A-S, Bergstraesser E, Grandjean C, Dorsaz A, Fahrni-Nater P, Cignacco E, et al. Comparison of end-of-life care practices between children with complex chronic conditions and neonates dying in an ICU versus non-ICUs: a substudy of the pediatric end-of-llfe care needs in Switzerland (PELICAN) Project. Pediatr Crit Care Med. 2020;21(5):e236-e46.

21. Agrawal R, Hall M, Cohen E, Goodman DM, Kuo DZ,NeffJM, et al.Trends in health care spending for children in Medicaid with high resource use. Pediatrics. 2016;138(4):e20160682.

22. Bernell S, Howard SW. Use your words carefully: what is a chronic disease? Public Health Front. 2016;4(159):1-3. 